Bulletin of the Section of Logic

Volume 46:1/2 (2017), pp. 11-19

http://dx.doi.org/10.18778/0138-0680.46.1.2.02

Ryszard Wójcicki

\title{
THREE DOCTRINES OF THE NATURE OF MATHEMATICS (SOME COMMENTS OF A KNOWLEDGE THEORIST)
}

\author{
Grzegorzowi, z serdecznymi życzeniami \\ i wyrazami wdziȩczności za wspieranie \\ moich planów i działań
}

\begin{abstract}
In this note I am reflecting on interrelations between three concepts of truth: (1) that employed by Hilbert arguing his formalist view on the nature of mathematics, (2) Freges idea of truth supported by mathematical intuition, and (3) known as Aristotelian correspondence idea of truth concerning any propositions not merely mathematical.
\end{abstract}

Keywords: mathematics, formalism, realism, intuitionism, truth

According to the Hilbert's doctrine of formalism, there are no principles that govern the processes of forming mathematical theories; mathematicians are free to form such theories in any way which, according to their judgments, results in revealing formal problems of considerable interest. The objects of mathematical analyses are determined by the theory's axioms abstract structures; the objectives of such analyses are learning properties of those structures. In a letter to Frege, who did not share Hilbert's formalist attitude Hilbert wrote:

You write: "I call axiom propositions that are true but are not proved because our knowledge of them flows from a source very different from the logical source, a source which might be called 
spatial intuition. From the truth of the axioms it follows that they do not contradict each other." I found it very interesting to read this sentence in your letter, for as long as I have been thinking, writing, and lecturing on these things, I have been saying the exact opposite: if the arbitrarily given axioms do not contradict each other with all their consequences, then they are true and the things defined by the axioms exist. For me this is the criterion of truth and existence ${ }^{1}$

Hilbert's and Frege's truth criteria of mathematical propositions are incompatible rather than inconsistent; they are related to different ideas of truth which thus can be referred to as $\operatorname{truth}_{H}$ and truth ${ }_{F}$, resp. Truth ${ }_{H}$ is a coherential notion of truth (for a formula, $p$, of a mathematical theory to be true, $p$ should be a deductive consequence of logically consistent system of axioms) $)^{2}$. Truth $F$ is defined in terms of the mathematician's "spatial intuition" a somewhat mysterious way of learning truth about something "behind of" the intuition. Truth $F$ appears to be alternative to $\operatorname{Truth}_{H}$ correspondence notion of truth; the correspondence between the examined formula $p$ and the determined by the mathematician' intuition physical (spatial) state of affair. One may wonder if it can be interpreted in this way indeed.

The fact that either scientists themselves, or offering their service mathematicians are able to make use of mathematics (selected parts of it) as the instrument of designing models of numerous natural and social phenomena is denied by no mathematician; mathematics is a powerful tool of handling questions specific to practically all fields of professional knowledge. The following issue, familiar as Königsberg bridge problem, exemplifies strong and direct relevance of mathematics to empirically decidable issues:

The Königsberg bridge problem asks if the seven bridges of the city of Königsberg [recently Kaliningrad] over the river Preger can all be traversed in a single trip without doubling back, with the additional requirement that the trip ends in the same

${ }^{1}$ Frege, G. 1980, Philosophical and Mathematical Correspondence. Ed. G. Gabriel, H. Hermes, F. Kambartel, C. Thiel, and A. Veraart. Abr. B. McGuinness and trans. H. Kaal. Chicago: University of Chicago Press, 39-40; quoted after Gregory H. Moore mcps.umn.edu/philosophy/11_4Moore.pdf.

${ }^{2}$ Some, not restricted to mathematics only variants of $\operatorname{truth}_{H}$ have been analyzed by R. Carnap and by K. Ajdukiewicz. 
place it began (see Fig. 1). [...] This problem was answered in the negative by Euler (1736), and represented the beginning of graph theory.

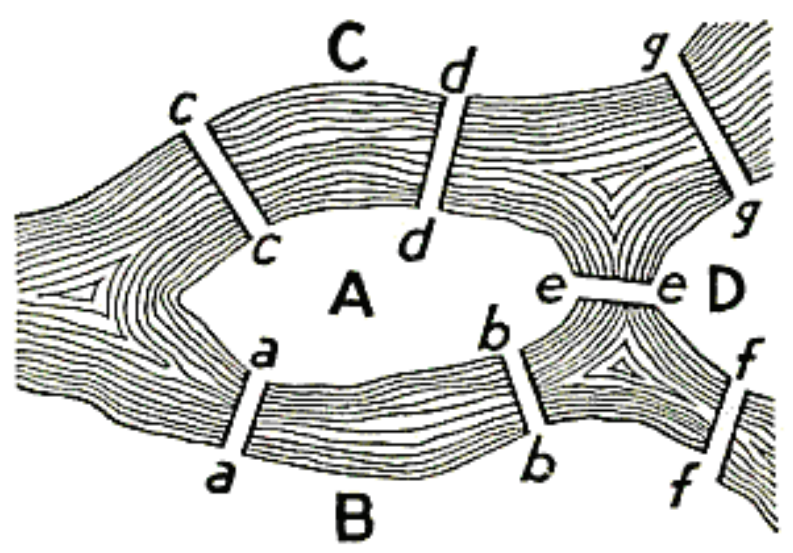

FIgURe 98. Geographic Map:

The Königsberg Bridges.

Fig. 1. Königsberg bridges (Quoted after http://mathworld.wolfram.com/ KoenigsbergBridgeProblem.html).

Apparently the field of interest of mathematicians engaged in the described problem was a factual situation. In fact, the factual state of affairs was of interest only as much as it satisfied the conditions defining the abstract construct corresponding to it. If the problem were examined as concerning physical reality, examining the possibility of traversing bridges over the river Preger should include any circumstances, e.g. flood that could make traversing some bridges impossible.

The abstract nature of the objects of mathematical investigations is not necessarily the outcome of shifting the interest in physical phenomena "such as they are" to their "idealized" (some philosophers prefer to say "counterfactual") counterparts. There is nothing "idealized" in Konigsberg's bridges and traversing them. What made them "abstract field of interest" was examining the bridge problem under the assumption that the 
bridges as the object of mathematical analyses will remain the same as they are at the time of defining with the problem; nothing destroy them or make non-traversable.

Realism as a view on the nature of mathematics differs radically from empirical realism being a view on the nature of knowledge conceived of as "empirically grounded" The most relax version of the latter is determined by several complementing each the other loose criteria. Of special relevance is the following:

ERC. Whatever can be confirmed empirically, practically unlimited number of times, by well-trained impartial observers or experimenters is likely to be real. So is whatever can be deduced (derived by truth-preserving reasoning) from the thus established "empirical facts".

Neither any single criterion nor any battery of criteria enables professional researchers to distinguish real things from appearances in a fully reliable manner. Fallibility of the idea of reality implies fallibility of that of truth. But - the point which has been argued by many thinkers (Dewey and Popper among others) - fallibility of our ideas of what is real and what is merely a product of our imagination does not imply that the ideas in question can never be correct. Likewise, fallibility of truth does not imply that we never can find out whether a state of affairs obtains or it does not.

In its outlined above the most permissive version empirical realism is compatible with the view that, besides physical bodies, the world is occupied by numerous mental (purely abstract included), cultural, legal, political ideas which, if internalized, manifest their existence modifying human behavior. By examining empirically consequences of internalized ideas one examines the ideas in question.

The proponents of intuitionism, the third doctrine on the nature of mathematics, are skeptical about the realist idea of truth accessibility. They are calling attention to the fact that numerous mathematical notions are neither related to any empirical phenomena nor even inspired by reflecting upon the phenomena of that kind. Such notions are outcomes of extrapolating mathematical ideas far beyond the limits of interest of the theorists using mathematics as an instrument of examining empirical phenomena. In this way mathematicians may arrive at conceptions leaving some issues relevant to them undecidable. As Feferman presenting his doctrine of "conceptual structuralism" puts it: "One may speak of what is true 
in a given conception, but that notion of truth may be partial. Truth in full is applicable only to completely definite conceptions." And he adds: What is clear in a given conception is time dependent, both for the individual and historically" 3 .

The presented above views on the nature of mathematics needs some additional comments. The formalist view is the outcome of skeptical attitude on the idea of tracing any "factual origins" of mathematical beliefs. For the theorist of professional knowledge adopting this view is tantamount of resigning from any attempt of accounting for fundamental relevance of mathematical ideas to factual knowledge development. The intuitionist does not deny that the idea of truth characteristic of mathematical analyses is grounded on the factual idea of truth. But, in distinction to the realist, the intuitionist tends to conceive mathematical truth as the property essentially different from its factual counterpart.

Besides defining differently the objects of the mathematician's analyses, the realist and the intuitionist disagree on which are the right criteria of soundness of mathematical reasoning. According to the former, the sufficient and necessary condition for a mathematical proof to be sound is "preserving truth;" if under some interpretation the premises of the mathematical argument are true, so has to be the conclusion. The intuitionist demands more: correct reasoning should not result in demonstrating the existence objects which one is not able to "construct effectively". For this reason, intuitionists "dislike" the set theoretic Axiom of Choice; it states the existence of sets for which there is no effective way of constructing them.

The development of mathematics is unlikely to depend on any way whatsoever to which of the outlined doctrines the mathematicians are ready to subscribe. Instead, the doctrines in question are of considerable interest for the theorist of knowledge rather than a mathematician.

The history of numerous mathematical theories, the oldest two - arithmetic of natural numbers and that of the Euclidean Geometry - included, exemplify the process of emerging "codified knowledge systems" from its "experiential" predecessors.

\footnotetext{
${ }^{3}$ Cf. Salomon Feferman, Logic, mathematics and conceptual structuralism, in The Metaphysics of Logic (P. Rush, ed.), Cambridge University Press (2014), 72-92. See also http://math.stanford.edu/ feferman/papers.html.
} 
By a "knowledge system" I'll mean a "relatively autonomous" branch of professional knowledge of any kind whatsoever. In case of mathematics, knowledge systems, besides taking the form of mathematical theories, may take the form of complexes of "mathematical units" related to specific mathematical problems and combining into conceptually coherent wholes: mathematical analysis, chaos theory, mathematical statistics, etc.

In turn, by codifying a knowledge system I mean setting the system's syntactical and semantic properties. Setting the former requires defining the language in which the specific to the system propositions (both statements and conjectures) are communicated. Setting the latter requires setting instructions enabling the users of the system to learn truth values of at least some of the system's propositions (recognize them as either true or false).

Arithmetic of natural numbers, AN, such as we know it nowadays has emerged from practical knowledge of counting objects whose scarcity or abundance might matter, and in particular knowledge of counting the outcomes of operations resulting in either increasing or decreasing the amount of such objects (the practical operations corresponding to adding and subtracting numbers). The origins of the Euclidean plain geometry, EG2, go back to practical abilities of handling topographical problems to which, among others, moving from one place to another or designing structures and roads connecting them on a limited area gives rise.

Roughly speaking, experiential knowledge is acquired by forming mental pictures, less or more exact, occasionally only schematized, of frequently encountered situations. Instances of thus acquired knowledge are referred to by cognitive psychologists as "cognitive schemata" or, if they concern some sequences of activities, "cognitive scripts". Neither AN nor EG2 in their experiential version reduce to a single cognitive schema. Rather they are numerous, preserved in the agents' minds collections of such, both discrete and generic, schemata of corresponding to the nature of these theories kinds.

With such schemata in the mind one does not needs to be familiar with any codified system of AN in order to count the number of items of a "collection" (a finite non empty set of objects), master the abstract operation of adding numbers by learning to count the elements of unified collections, and master subtracting numbers by learning to count the elements of collections separated as parts of some larger ones. The way in which EG2 is related to its experiential origins is similar. 
To account of the mental processes that eventually resulted in emerging the codified version of AN and EG2 is the task of cognitive scientists. But accounting of the codification procedures is the task of metamathematicians. In two provided below subsections I am discussing some selected aspects of the former.

Euclidean plain geometry EG2. Euclid's codification of the two dimensional geometry has been restricted to setting the axiom system that as Euclid expected - should enable its properly trained users to derive by truth preserving reasoning all theses which one is able to identify as true by reflecting on the held in mind geometric ideas (in fact, the variety of topographic schemas). The minimal requirement that properly done codification of an axiom system should satisfy is distinguishing clearly primitive terms appearing in axiom from the terms determining the "deductive apparatus" of the theory. The axioms are postulated to determine the meanings (legitimate usages) of the primitive terms, while the meanings of terms specific to deductive apparatus should be determined by the valid in the system inference instructions.

The set by Euclid list of the primitive terms consisted of: points, straight lines and their segments determined by points located in them, distances between two points located on the same line (length of a line segment), angles between two crossing themselves lines, circles determined by their centers and distances for their radiuses. No list of terms that suffice to codify the deductive features of EG2 was given; that part of EG2 was left non-codified. It does not mean, however, that the experiential characteristic of it was unclear. Besides its logical part being the same as in numerous others cases of reasoning which ancient philosophers employed in its experiential version on numerous occasions, it involved the experiential idea of moving geometrical figures in the way preserving their metric properties (distances of located on them points and angles between crossing lines).

Euclidean axioms tell nothing about the operations in question; one learns them from supplementing the axioms informal comments. But experiential ideas of these operations are as essential complement of the codified version of EG2 as the Euclid's axioms. By performing those operations one may (1) construct new geometric lines and figures thus forming definitional extensions of EG2 and (2) measure distances and angles and thus to produce deductive argument involving these concepts. The anal- 
ysis of the problems to which the task of codifying these operations gave rise was provided by Felix Klein in Vergleichende Betrachtungen ber neuere geometrische Forschungen, Verlag von Andreas Deichert, Erlangen, 1872.

The very long - lasting many centuries - process of codifying predicate which in its tacit form is traceable in mathematical reasoning, from the earliest days of people's struggling with mathematical issues was completed late in the nineteenth century when as Gregory H. Moore ${ }^{4}$ put it "the notion of quantifier was disentangled from the notion of prepositional connective and was given an appropriate symbolic representation. These two developments were both brought about independently by two mathematicians having a strong philosophical bent-Charles Sanders Peirce and Gottlob Frege".

Peano Arithmetic AN. G. Peano's (1889) system of five axioms involving three primitive terms: $N$ (the set of all natural numbers), $\mathrm{S}$ (successor operation), and 0 (the least natural number). The axioms (A1) $0 \in N$, (A2) $n \in N \rightarrow S n \in N$, (A3) $n \in N \rightarrow S n \neq 0$, and (A4) $\left(n \in N \wedge n^{\prime} \in\right.$ $\left.N \wedge S n=S n^{\prime}\right) \rightarrow n=n^{\prime}$, and the axiom of induction which, equivalently to its original version, can be stated as the following instruction of recursive reasoning: Let $\mathrm{P}$ be a unary predicate, then

$$
0 \in P, \forall n(n \in P \rightarrow S n \in P) \vdash N \subseteq P
$$

As in any case of codifying experiential beginning of a mathematical theory, Peano AN gives rise to the question of completeness of the offered codification. All Peano's axioms are "intuitively true." Nothing suggests that they may be logically inconsistent, either. Suppose one decides to take for granted that the Peano axioms are both true $F$ and true . $_{\text {. }}$

As Gödel has succeeded to prove, by establishing his famous Incompleteness Theorem, neither Peano's axiom system, nor any improved version of it can be complete; AN cannot be codified in the way assuring that every true proposition expressed in the language in which AN has been codified is either an axiom or is deductively derivable from the axioms, characterising the codification. Moreover, it is so regardless of whether the axiom system in question, concerns arithmetic operations exclusively, or any additional besides them.

\footnotetext{
${ }^{4}$ See: mcps.umn.edu/philosophy/11_4Moore.pdf.
} 
Which of two, true $F$ and true $H$, concepts of truth does Gödel's Incompleteness Theorem refer to? The right answer is: both. The provided by Gödel proof of the discussed theorem hinges on two assumptions: (1) deductive reasoning preserves truth $\mathrm{t}_{H}$, and (2) whichever criteria of truth one is ready to follow, in order for a proposition $\mathrm{p}$ to be true in virtue of the criteria in question, $p$ has to be true ${ }_{H}$ (the adopted criteria of truth cannot be inconsistent with criteria of "logical truth").

The relevance of Gödel's incompleteness theorem for consideration concerning any knowledge systems, not merely mathematical theories is obvious. In their rigorously codified versions, all such systems become pieces of "applied mathematics". As such they cannot represent AN as auxiliary systems and consequently they are incomplete.

\section{References}

[1] S. Feferman, Logic, mathematics and conceptual structuralism, [in:] The Metaphysics of Logic (P. Rush, ed.), Cambridge University Press (2014), pp. 72-92.

[2] G. Frege, Philosophical and Mathematical Correspondence, ed. G. Gabriel, H. Hermes, F. Kambartel, C. Thiel, and A. Veraart. Abr. B. McGuinness and trans. H. Kaal. Chicago: University of Chicago Press, 1980, pp. 39-40.

[3] F. Klein, Vergleichende Betrachtungen ber neuere geometrische Forschungen, Verlag von Andreas Deichert, Erlangen, 1872.

\section{IFIS PAN}

e-mail: rw.mazury@gmail.com 